\title{
A Importância da Atuação do Nutricionista na Atenção Básica à Saúde
}

\section{The Importance of the Nutritional Performance in Primary Care}

Priscila Fonte Mattos ${ }^{1}$

Alden dos Santos Neves ${ }^{2}$

\section{Resumo}

Ultimamente tem ocorrido no Brasil uma acelerada transição nutricional que influenciou o perfil epidemiológico causando mudanças nos padrôes de morbimortalidade, conseqüentemente gerando aumento da obesidade e doenças crônicas não transmissíveis. Para a resolução desse problema torna-se necessário um modelo de atenção básica à saúde com os princípios de universalidade, integralidade e equidade, tendo suas práticas voltadas para a vigilância à saúde, principalmente no que diz respeito à segurança alimentar. O presente trabalho propóe uma reflexão sobre a importância da atuação do nutricionista na atenção básica. O objetivo é apresentar argumentos que mostrem que é indispensável a presença do nutricionista no Estratégia Saúde da Família e Núcleo de Apoio ao Saúde da Família. Sendo assim, a inserçâo do profissional nutricionista na Atenção Básica como no Programa de Saúde da Família torna-se indispensável para a mudança do quadro nutricional no Brasil. Com a criação do Núcleo de Apoio à Saúde da Família se torna mais claro o papel do nutricionista na promoção da saúde, pois o NASF veio por em prática o compromisso da integração de alimentação e nutrição com o setor saúde para melhorar a qualidade da Atenção Básica.

Pavavras-chave: Nutricionista; Segurança Alimentar e Nutricional; Atenção Básica.

\section{Abstract}

Lately there has been in Brazil a rapid nutritional transition that influenced the epidemiological profile causing changes in the patterns of morbidity and mortality, thus generating an increase in obesity and chronic diseases. To solve this problem it is necessary a model of primary health care with the principles of universality, comprehensiveness, and equity, with its practices for the health surveillance, particularly with regard to food and nutritional security. This paper proposes a reflection on the importance of the nutritionist's role in primary care. The goal is to present arguments to show that it is essential the presence of the nutritionist in the Family's Health Strategy and Support Center for Family's Health. Thus, the insertion of a professional nutritionist in Primary Care Program and Family Health is indispensable to change the nutritional status in Brazil. The creation of the Support Center for Family Health becomes clearer the role of nutritionists in health promotion, as the Nasf came into practice the commitment of integrating food and nutrition to the health sector to improve the quality of primary care.

Keywords: Nutritionist; Primary Health Care; Family Health Program.

${ }^{1}$ Nutricionista graduada pelo Centro Universitário de Volta Redonda (UniFOA) - Volta Redonda/RJ ${ }^{2}$ Professor do Curso de Nutriçāo do Centro Universitário de Volta Redonda (UniFOA)-Volta Redonda/RJ, Mestrando em Ensino de Ciências da Saúde e Meio Ambiente pelo Centro Universitário de Volta Redonda(UniFOA) 


\section{INTRODUÇÃO}

O Brasil apresentou nos últimos anos uma rápida transição demográfica, epidemiológica e nutricional, e tem chamado atenção o aumento da obesidade em diversos grupos populacionais. Sendo assim a obesidade se consolidou como agravo nutricional e, associado a uma alta incidência de doenças crônicas não transmissíveis como diabetes, câncer e doenças cardiovasculares, influencia o perfil epidemiológico, causando mudanças nos padróes de morbimortalidade (MELÉNDEZ, 2003).

O consumo inadequado de alimentos, com alta ingestão de produtos pouco saudáveis ou até mesmo a falta de alimentos gera a insegurança alimentar, que no Brasil está associada à falta de acesso, que teria de ser o direito de cada cidadão. Por esta condição de insegurança alimentar, gerando sobrepeso, obesidade, desnutrição e doenças crônicas, é indispensável um modelo de atenção à saúde no âmbito do Sistema Único de Saúde (SUS), que associe esses aspectos para uma intervenção adequada.

Nos dias atuais, a busca da integralidade é fundamental para a formação de cada profissional da área da saúde. A Estratégia de Saúde da Família (ESF) pauta-se nos princípios do SUS de universalidade, integralidade e equidade, tendo suas práticas voltadas para a vigilância à saúde. Sendo assim, a inserção do profissional nutricionista na atenção básica à saúde, especificamente no Programa de Saúde da Família (PSF) torna-se necessário para a resoluçáo de problemas alimentares e prevenção de doenças causadas pela insegurança alimentar. Além de fazer a prevenção dessas doenças através da atenção primária à saúde, a atuação do profissional nutricionista na Atenção Básica faz com que haja uma recepção imediata e humanizada, buscando muitas vezes a solução imediata do problema ou a definição do melhor encaminhamento para sua resolução (PINHEIRO, 2008).

O PSF visa substituir o modelo tradicional médicocentrado para um novo modelo de integração interdisciplinar, com a intenção de reorganizar a prática da atenção à saúde. Com isso, o profissional de nutrição tem o importante papel de promover uma reeducação dos hábitos alimentares da população fazendo a prevenção de doenças e a promoção da saúde através da qualidade de vida (SANTOS, 2005).

Segundo Amoretti (2005), a presença de vários especialistas trabalhando no PSF prejudica o processo da assistência, basicamente porque esses especialistas não foram preparados para esse tipo de trabalho, e que muitas vezes o encaminhamento para outras especialidades são desnecessários e inadequados. Tal afirmação contradiz o propósito do PSF, que é o de prestar assistência mais completa à população a partir de açóes voltadas para uma atenção primária através de equipe multiprofissional proporcionando um atendimento mais qualificado e dentro do principio da integralidade (AMORETTI, 2005; SORATTO, 2005).

De acordo com o estudo feito por Santos (2005), médicos e enfermeiros falam das dificuldades que possuem para orientar seus pacientes sobre alimentação e relatam também que não têm formação adequada na área de nutrição, sendo esses os profissionais que dão orientações dietéticas quando não há um nutricionista no PSF.

O foco no tratamento curativo exclusivamente encontrase atualmente fora dos propósitos das políticas públicas em saúde, pois é mais vantajoso para os gestores e para a população o foco em prevenção e promoçáo de saúde, especialmente através da mudança no estilo de vida, com alteraçôes em hábitos alimentares e de atividade física (SANTOS, 2005).

A educação em saúde faz parte das atribuiçóes de qualquer profissional que integre a equipe do PSF. O profissional de nutrição, além da prescrição dietética, precisa transmitir informaçóes sobre hábitos saudáveis. Essa prática faz com que se desenvolva a autonomia diante do conhecimento do processo saúde-doença (ALVES; NUNES,2006).

Em janeiro de 2008 entrou em vigor o Núcleo de Apoio à Saúde da Família (NASF), criado pelo Ministério da Saúde através da portaria GM n ${ }^{\circ} 154$, de 24 de janeiro de 2008, para melhorar a qualidade da atenção básica à saúde através de equipes compostas por profissionais de diferentes áreas de conhecimento, dentre eles o nutricionista, contribuindo com a promoção de práticas alimentares saudáveis.

O NASF vem pôr em prática o compromisso da integração de alimentação e nutrição com o setor saúde, com relação aos componentes de Segurança Alimentar e Nutricional visando o direito humano à alimentação adequada, através da inserção do nutricionista na Atenção Básica à Saúde.

O presente trabalho visa demonstrar a importância da atuação do nutricionista na atenção básica à saúde devido à transição nutricional e ao compromisso que as açóes de alimentação e nutrição têm com a saúde pública. O objetivo é apresentar argumentos que mostram a importância do profissional nutricionista na área da atenção básica à saúde auxiliando sua inserção no PSF e NASF, e na área da educação para saúde enquanto promotor de saúde.

\section{TRANSIÇÃO NUTRICIONAL NO BRASIL}

O Brasil tem vivido nos últimos anos uma acelerada transição demográfica, epidemiológica e nutricional. A transição demográfica é caracterizada pelo ritmo de crescimento populacional provocado por mudanças nos níveis de fecundidade, natalidade e mortalidade. Já a transição epidemiológica é caracterizada por mudanças nos padróes de morbimortalidade relacionados principalmente à reduçáo 
da mortalidade precoce, aumento da expectativa de vida ao nascer tornando a população mais idosa, processo intenso de urbanização e mudanças socioculturais. O conceito de transição nutricional, que se configura dentro do modelo de transição epidemiológica, refere-se a mudanças nos padróes de nutrição, devido à modificação da alimentação, conseqüente de transformaçôes demográficas e epidemiológicas (OLIVEIRA, 2004).

Mais especificamente, entende-se por Transição $\mathrm{Nu}$ tricional a mudança nos padróes de problemas nutricionais de uma população, em geral uma mudança da desnutrição para a obesidade. $\mathrm{O}$ aumento da obesidade está presente em diversos grupos populacionais apresentando um agravo nutricional associado a doenças crônicas não transmissíveis, como doenças cardiovasculares, câncer e diabetes (KAC; MELÉNDEZ, 2003).

As transiçôes demográfica, epidemiológica e nutricional manifestam-se em conjunto devido à agravos de saúde resultantes do mesmo problema, que é a alimentação inadequada e má nutrição. A presença de desnutrição, obesidade, anemia e diabetes, principalmente numa mesma família, comprovam a insegurança alimentar e nutricional que no Brasil está associada a negação do direito ao acesso a alimentação necessária à vida e alimentação inadequada (PINHEIRO, 2008).

\section{ATENÇÃO BÁSICA}

Apesar do crescimento da obesidade, os problemas relativos à deficiência de acesso a alimentação qualitativa ou quantitativamente adequadas causa ainda problemas de desnutrição ou carência de micronutrientes. Estudos demonstram que uma criança que apresenta desnutrição na infância tem um risco bem mais elevado de desenvolver doenças crônicas não transmissíveis, como a obesidade, na fase adulta, do que crianças eutróficas. Cerca da metade do total de óbitos da população brasileira são associados a doenças crônicas não-transmissíveis (PINHEIRO, 2008).

Considerando esses fatos, se torna necessário uma reorganização do modelo de atenção básica à saúde, no âmbito do Sistema Único de Saúde. Recentemente, as açóes relativas à atenção básica tem se concentrado no processo de expansão do Programa Saúde da Família (PSF), incentivado pelo Ministério da Saúde (MARQUES; MENDES, 2002).

O Programa de Saúde da Família (PSF) foi criado pelo Ministério da Saúde em 1994, com o propósito de assumir a reorganização da atenção básica à saúde, ou seja, substituir o modelo tradicional por outro com novas práticas e equipe multiprofissional, garantindo à população brasileira uma oferta de serviços contínuos pelos profissionais que com- póem as equipes do PSF seja na Unidade básica de saúde ou em domicílio, bem como o fortalecimento dos princípios de universalidade, acessibilidade, integralidade e equidade do SUS.

Pesquisas realizadas sobre o perfil de recursos humanos no PSF demonstraram que há na composição das equipes o mínimo recomendado pelo Ministério da Saúde, o que mostra o cumprimento da lei. Porém, com relação à participação de outros profissionais de saúde nas equipes de saúde da família, verificou que estes são muito reduzidos, sendo o nutricionista o que menos aparece (SANTOS, 2005; SORATTO, 2005).

De acordo com o Conselho Federal de Nutricionistas (2005), compete ao nutricionista, na área de Saúde Coletiva, prestar assistência e educação nutricional para a comunidade. E para realizar essas atribuiçóes, precisa participar de equipes multiprofissionais entre outras atividades. O PSF é um programa institucional que abre as portas para essa atuação do nutricionista na área de saúde coletiva. Porém, em muitos PSFs ainda há ausência de nutricionista (CFN, 2005).

\section{A IMPORTÂNCIA DO NUTRICIONISTA NA ATENÇÃO BÁSICA}

Um estudo feito por Santos (2005) que utilizou a opinião de diferentes trabalhadores da área de saúde sobre a inserção do nutricionista no PSF, relatou que profissionais da saúde passam por problemas relacionados à falta do nutricionista e usam argumentos que auxiliam nessa integração como dúvidas e falta de conhecimento para responder questôes relacionadas à nutrição.

O nutricionista é o único profissional que recebe na sua formação acadêmica um conhecimento específico que lhe permite a partir de diagnóstico e observação de valores socioculturais de cada paciente, propor as devidas orientaçôes nutricionais adequando-as à realidade de cada família, sendo um profissional indispensável no modelo de atenção à saúde proposto pelo governo (SANTOS, 2005).

O profissional de nutrição tem o importante papel de promover uma reeducação dos hábitos alimentares da população fazendo a prevenção de doenças e a promoção da qualidade de vida. Nos serviços de saúde, através das consultas com monitoramento do estado nutricional, há possibilidade de diagnósticos longitudinais, o que é de grande importância para a melhoria das condiçóes alimentares e nutricionais da comunidade. Com a coleta de dados antropométricos integrado a outros marcadores de saúde como hipertensão e diabetes, há uma resposta mais efetiva com relação à necessidade de cada paciente ( MACHADO, 2006). 


\section{EDUCAÇÃO EM SAÚDE}

Uma das atribuiçóes dos profissionais que fazem parte das equipes do PSF é a Educação em Saúde. Além de formar grupos educativos sobre patologias específicas, os profissionais devem sempre abordar o assunto saúde em suas consultas através de orientaçóes adequadas, cabendo ao nutricionista oferecer informaçôes sobre uma boa alimentação para a prevenção de doenças (ALVES; NUNES, 2006).

A educação em saúde proposta pelo PSF contribui para estimular a responsabilidade comunitária e a busca por melhores condições de vida. Além de orientar a população sobre a prevenção de doenças, estimula uma aproximação entre o conhecimento popular e o científico. Compreender o conhecimento popular torna-se indispensável para a exposição do conhecimento científico de maneira aceitável, por isso é muito importante entender como as pessoas pensam e praticam a saúde e, a partir daí, adequar os bons hábitos alimentares às necessidades da comunidade (GOTTARDI; SILVA, 2005).

$\mathrm{O}$ atendimento nutricional prestado pelo profissional nutricionista mostra a importância da sua atuação na atenção básica à saúde. Isso tem permitido uma aproximação com os outros profissionais que atuam nas Unidades de Saúde aprofundando a questão alimentar familiar através de troca de informaçôes. Esse espaço educativo que é o atendimento individual à saúde, permite também vínculos com os indivíduos e seus familiares, compartilhando a responsabilidade de promoção e manutenção da saúde (PINHEIRO, 2008).

\section{NASF}

Para fortalecer a Estratégia Saúde da Família e melhorar a qualidade da atenção básica à saúde, o Ministério da Saúde criou os Núcleos de Apoio à Saúde da Família (NASF) pela portaria $n^{\circ} 154$, de 24 de janeiro de 2008. A proposta da criaçáo do NASF teve como objetivo aumentar a abrangência das açôes de atenção básica à saúde. Os profissionais do NASF devem atuar em parceria com os profissionais das equipes de Saúde da Família. Os núcleos do NASF são compostos por no mínimo cinco profissionais, dentre várias ocupaçóes, sendo um deles o nutricionista.

Algumas açóes de alimentação e nutrição propostas pelo NASF são conhecer e estimular o consumo dos alimentos saudáveis produzidos regionalmente; capacitar a equipe de saúde da família e participar de açôes vinculadas aos programas de controle e prevenção dos distúrbios nutricionais; elaborar em conjunto com as equipes de saúde da família rotinas de atenção nutricional e atendimento para doenças relacionadas à Alimentação e Nutrição, de acordo com os protocolos de atenção básica.
Sendo assim, as açôes de Alimentação e Nutrição integram o compromisso da Atenção Básica, assumindo o papel de fazer a prevenção, promoção e recuperação da saúde através de práticas alimentares saudáveis.

\section{CONSIDERAÇÓES FINAIS:}

No campo da nutrição, a transição epidemiológica representa mudanças no perfil de morbimortalidade devido à modificação de hábitos alimentares, que gera o aumento da obesidade e doenças crônicas náo transmissíveis. E as açóes do setor saúde ainda não apresentam um grau de agilidade suficiente para responder aos desafios conseqüentes da mudança do quadro epidemiológico.

A atual situação epidemiológica nutricional exige a prática dos princípios da integralidade, equidade e universalidade na assistência à saúde, dando importância aos estudos que acompanhem a evolução nutricional da população, que são de significativa importância, pois colocam em prática políticas públicas de Segurança Alimentar e Nutricional, transformando em realidade o direito humano à alimentação adequada.

A Estratégia de Saúde da Família e NASF são campos de atuaçáo muito importantes para garantir a Segurança Alimentar e Nutricional no Brasil. O controle e tratamento dos agravos à saúde estão relacionados claramente com a alimentação da população, seja diretamente ou indiretamente, ressaltando a importância do nutricionista na Atenção Básica.

O profissional nutricionista possui conhecimentos capazes de melhorar o perfil epidemiológico e nutricional da população. A falta do nutricionista abre espaço para que outros profissionais façam uso, de forma superficial, de seus conhecimentos sobre alimentação e nutrição, mesmo sabendo que não possuem formação adequada nessa área.

Por esse motivo e vários outros já expostos, a inserção do nutricionista na Atenção Básica é de suma importância, pois o modelo proposto pelo governo não é apenas recuperar a saúde após a instalação da doença, mas também prevenir doenças e promover a saúde, o que se consegue através de uma alimentação adequada.

A partir do discutido anteriormente pode-se concluir que a participação do nutricionista e sua integração na saúde básica está estabelecida em sua formação acadêmica que o capacita a realizar diagnóstico nutricional da população, tornando-o o único profissional a receber um conhecimento específico que lhe pemita propor as devidas orientaçóes dietéticas, a partir de diagnóstico e da observação dos valores socioculturais, adequando-as à realidade de cada unidade familiar. Portanto, um profissional apto a reafirmar o modelo de atenção à saúde proposto em nosso país. 


\section{REFERÊNCIAS:}

PINHEIRO, A. R. O. et al. Nutrição em saúde Pública: Os potenciais de inserçáo na Estratégia de Saúde da Família (ESF). Revista eletrônica Tempus. Vol. 1, No 1, 2008. Disponível em: http://164.41.105.3/portalnesp/ojs2.1.1/index.php/tempus

OLIVEIRA, R. C. A transiçáo nutricional no contexto da transiçáo demográfica e epidemiológica. Rev. Min. Saúde Pública, v.3, n.5, p.16-23 - Jul./dez.2004.

MELÉNDEZ, G. V.; KAC G. A transiçáo nutricional e a epidemiologia da obesidade na América Latina. Cad. Saúde Pública, Rio de Janeiro, 19(Sup. 1):S4-S5, 2003.

MARQUES, R. M.; MENDES, A. A política de incentivos do Ministérioda Saúde para a atençáo básica: uma ameaça à autonomia dos gestoresmunicipais e ao princípio da integralidade? Cad. Saúde Pública, Rio de Janeiro, 18(Suplemento):163-171, 2002.

SANTOS, A. C. A inserçáo do nutricionista na estratégia da saúde da família: o olhar de diferentes trabalhadores da saúde. Fam. Saúde Desenv., Curitiba, v.7, n.3, p.257265, set./dez. 2005.

SORATTO, E. O que é PSF? Florianópolis: Centro Acadêmico Livre de Enfermagem, 2005.

CFN - CONSELHO FEDERAL DE NUTRICIONISTAS. Resolução CFN nº 380/2005.

MACHADO, N. M. V. et al. Reflexóes sobre saúde, nutriçáo e a estratégia de saúde da família. 2006. Disponível em: http://dtr2004.saude.gov.br/nutricao/documentos/ noticia_01_09_06.pdf.

Acessado em 17abril2008.

SILVA, A. K. et al. A inserçáo do nutricionista no programa saúde da família (PSF). III Encontro de Pesquisa de IES do Sistema Estadual de Minas Gerais. UNEC - Caratinga/MG.2006.

ALVES, V. S.; NUNES, M. O. Educaçáo em Saúde na atençáo médica ao paciente com hipertensáo arterial no Programa Saúde da Família. Interface - Comunic, Saúde, Educ, v.9, n.18, p.131-47, jan/jun 2006.
AMORETTI, R. A Educaçáo Médica diante das Necessidades Sociais em Saúde. Rev Bras. Educ. Méd. Rio de Janeiro, v .29, nº 2, p.136-146, maio/ago. 2005.

GOTTARDI, R. L. B.; SILVA, A. L. Educaçáo em Saúde: o agente comunitário de saúde sob foco. Boletim do Instituto de Saúde, n..37, p.10-12, dezembro de 2005.

BRASIL. Ministério da Saúde. Portaria no 154., 24 de janeiro de 2008. Trata dos Núcleos de Apoio à Saúde da Família - NASF.
Endereço para Correspondência:

Alden dos Santos Neves alden.neves@foa.org.br

Mestrado Profissional em Ensino em Ciências da Saúde e do Meio Ambiente

Centro Universitário de Volta Redonda Campus Três Poços Av. Paulo Erlei Alves Abrantes, no 1325 , Três Poços - Volta Redonda / RJ CEP: 27240-560 